\title{
On Common Fixed Point for Single and Set-Valued Maps Satisfying OWC Property in IFMS using Implicit Relation
}

\section{Jong Seo Park}

Department of Mathematics Education, Chinju National University of Education, Jinju, Korea

\section{ljfis}

Received: Jan. 27, 2015

Revised : May 25, 2015

Accepted: May 27, 2015

Correspondence to: Jong Seo Park

(parkjs@cue.ac.kr)

(CThe Korean Institute of Intelligent Systems

cCThis is an Open Access article distributed under the terms of the Creative Commons Attribution Non-Commercial License (http://creativecommons.org/licenses/ by-nc/3.0/) which permits unrestricted noncommercial use, distribution, and reproduction in any medium, provided the original work is properly cited.

\begin{abstract}
In this paper, we introduce the notion of single and set-valued maps satisfying OWC property in IFMS using implicit relation. Also, we obtain common fixed point theorems for single and set-valued maps satisfying OWC properties in IFMS using implicit relation.
\end{abstract}

Keywords: Common fixed point, Occasionally weakly compatible map, Implicit relation.

\section{Introduction}

Several authors [1-5] studied and developed the various concepts in different direction and proved some fixed point in fuzzy metric space. Also, Jungck [6] introduced the concept of compatible maps, and Vijayaraju and Sajath [7] obtained some common fixed point theorems in fuzzy metric space. Recently, Park et.a.. [8] introduced the intuitionistic fuzzy metric space (IFMS), Park [12, 13] studied the compatible and weakly compatible maps in IFMS, and proved common fixed point theorem in IFMS. Also, Park [9] proved some properties for several types compatible maps, and Park [10] defined occasionally weakly semi-compatible map and obtained some fixed point using this maps in IFMS.

In this paper, we introduce the notion of single and set-valued maps satisfying occasionally weakly compatible (OWC) property in IFMS using implicit relation. Also, we obtain common fixed point theorems for single and set-valued maps satisfying OWC property in IFMS using implicit relation.

\section{Preliminaries}

In this part, we recall some definitions, properties and known results in the IFMS as follows : Let us recall ( [11]) that a continuous $t$-norm is an operation $*:[0,1] \times[0,1] \rightarrow[0,1]$ which satisfies the following conditions: (a) $*$ is commutative and associative, (b) $*$ is continuous, (c) $a * 1=a$ for all $a \in[0,1]$, (d) $a * b \leq c * d$ whenever $a \leq c$ and $b \leq d(a, b, c, d \in[0,1])$. Also, a continuous $t$-conorm is an operation $\diamond:[0,1] \times[0,1] \rightarrow[0,1]$ which satisfies the following conditions: (a) $\diamond$ is commutative and associative, (b) $\diamond$ is continuous, (c) $a \diamond 0=a$ for all $a \in[0,1],(\mathrm{d}) a \diamond b \geq c \diamond d$ whenever $a \leq c$ and $b \leq d(a, b, c, d \in[0,1])$.

Definition 2.1. ([8]) The 5 -tuple $(X, M, N, *, \diamond)$ is said to be an intuitionistic fuzzy metric space (IFMS) if $X$ is an arbitrary set, $*$ is a continuous $t$-norm, $\diamond$ is a continuous $t$-conorm and $M, N$ are fuzzy sets on $X^{2} \times(0, \infty)$ satisfying the following conditions; for 
all $x, y, z$ in $X$ and all $s, t \in(0, \infty)$,
(a) $M(x, y, t)>0$,
(b) $M(x, y, t)=1$ if and only if $x=y$,
(c) $M(x, y, t)=M(y, x, t)$,
(d) $M(x, y, t) * M(y, z, s) \leq M(x, z, t+s)$,
(e) $M(x, y, \cdot):(0, \infty) \rightarrow(0,1]$ is continuous,
(f) $N(x, y, t)>0$,
(g) $N(x, y, t)=0$ if and only if $x=y$,
(h) $N(x, y, t)=N(y, x, t)$,
(i) $N(x, y, t) \diamond N(y, z, s) \geq N(x, z, t+s)$,
(j) $N(x, y, \cdot):(0, \infty) \rightarrow(0,1]$ is continuous.

Note that $(M, N)$ is called an IFM on $X$. The functions $M(x, y, t)$ and $N(x, y, t)$ denote the degree of nearness and the degree of non-nearness between $x$ and $y$ with respect to $t$, respectively

Through out this paper, $X$ will represent the IFMS and $C B(X)$, the set of all non-empty closed and bounded subsets of $X$. For $A, B \in C B(X)$ and for every $t>0$, denote

$$
\begin{aligned}
& H(A, B, t)=\sup \{M(a, b, t) ; a \in A, b \in B\}, \\
& h(A, B, t)=\inf \{N(a, b, t) ; a \in A, b \in B\}, \\
& \delta_{M}(A, B, t)=\inf \{M(a, b, t) ; a \in A, b \in B\}, \\
& \delta^{N}(A, B, t)=\sup \{N(a, b, t) ; a \in A, b \in B\} .
\end{aligned}
$$

If $A$ consists of a single point $a$, we write

$$
\delta_{M}(A, B, t)=\delta_{M}(a, B, t), \quad \delta^{N}(A, B, t)=\delta^{N}(a, B, t) .
$$

Furthermore, if $B$ consists of a single point $b$, we write

$$
\delta_{M}(A, B, t)=M(a, b, t), \quad \delta^{N}(A, B, t)=N(a, b, t) .
$$

It follows immediately from definition that

$$
\begin{aligned}
& \delta_{M}(A, B, t)=\delta_{M}(B, A, t) \geq 0, \\
& \delta^{N}(A, B, t)=\delta^{N}(B, A, t) \leq 1 .
\end{aligned}
$$

Also, $\delta_{M}(A, B, t)=1$ and $\delta^{N}(A, B, t)=0$ if and only if $A=B=\{a\}$ for al $A, B \in C B(X)$.

Definition 2.2. Let $X$ be an IFMS, $A: X \rightarrow X$ and $B: X \rightarrow$ $C B(X)$.

(a) A point $x \in X$ is called a coincidence point of hybrid maps $A$ and $B$ if $x=A x \in B x$.

(b) Hybrid maps $A$ and $B$ are said to be compatible if $A B x \in$
$C B(X)$ for all $x \in X$ and

$$
\begin{aligned}
& \lim _{n \rightarrow \infty} H\left(A B x_{n}, B A x_{n}, t\right)=1, \\
& \lim _{n \rightarrow \infty} h\left(A B x_{n}, B A x_{n}, t\right)=0
\end{aligned}
$$

whenever $\left\{x_{n}\right\}$ is a sequence in $X$ such that $B x_{n} \rightarrow D \in$ $C B(X)$ and $A x_{n} \rightarrow x \in D$.

(c) Hybrid maps $A$ and $B$ are said to be weakly compatible if $A B x=B A x$ whenever $A x \in B x$.

(d) Hybrid maps $A$ and $B$ are said to be occasionally weakly compatible (OWC) if there exists some points $x \in X$ such that $A x \in B x$ and $A B x \subseteq B A x$.

Example 2.3. Let $X=[0, \infty)$ with $a * b=\min \{a, b\}, a \diamond b=$ $\max \{a, b\}$ for all $a, b \in[0,1]$ and for all $t>0$,

$$
M(x, y, t)=\frac{t}{t+d(x, y)}, N(x, y, t)=\frac{d(x, y)}{t+d(x, y)} .
$$

Define the maps $A: X \rightarrow X$ and $B: X \rightarrow C B(X)$ by

$$
\begin{aligned}
& A x= \begin{cases}0 & \text { if } 0 \leq x<1, \\
x+1 & \text { if } 1 \leq x<\infty,\end{cases} \\
& B x= \begin{cases}\{0\} & \text { if } 0 \leq x<1, \\
{[1, x+3]} & \text { if } 1 \leq x<\infty .\end{cases}
\end{aligned}
$$

Here 1 is a coincidence point of $A$ and $B$, but $A$ and $B$ are not weakly compatible as $B A(1)=[1,5] \neq A B(1)=[2,5]$ Also, $A$ and $B$ are OWC hybrid maps as $A$ and $B$ are weakly compatible at $x=0$ as $A(0) \in B(0)$ and $0=A B(0) \subseteq$ $B A(0)=\{0\}$. Hence weakly compatible hybrid maps are OWC, but the converse is not true in general.

\section{Main Results}

Theorem 3.1. Let $X$ be an IFMS with $t * t=t$ and $t \diamond t=t$ for all $t \in[0,1]$. Also, let $A, B: X \rightarrow X$ and $S, T: X \rightarrow$ $C B(X)$ be single and set-valued mappings such that the hybrid pairs $(A, S)$ and $(B, T)$ are OWC satisfying

$$
\begin{gathered}
\phi\left\{\delta_{M}(S x, T y, t), M(A x, B y, t),\right. \\
H(A x, S x, t), H(B y, T y, t), \\
H(A x, T y, t) * H(B y, S x, t)\} \geq 0 \\
\psi\left\{\delta^{N}(S x, T y, t), N(A x, B y, t),\right. \\
h(A x, S x, t), h(B y, T y, t), \\
h(A x, T y, t) \diamond h(B y, S x, t)\} \leq 1
\end{gathered}
$$


for every $x, y \in X, t>0$.

Also, let implicit relation $\Phi=\{\phi, \psi\}$ such that $\phi:[0,1]^{5} \rightarrow$ $[0,1]$ and $\psi:[0,1]^{5} \rightarrow[0,1]$ continuous functions satisfying

(a) $\phi\left(t_{1}, t_{2}, t_{3}, t_{4}, t_{5}\right)$ is non-increasing in $t_{2}$ and $t_{5}$ for all $t>0$. $\psi\left(t_{1}, t_{2}, t_{3}, t_{4}, t_{5}\right)$ is non-decreasing in $t_{2}$ and $t_{5}$ for all $t>0$.

(b) $\phi(t, t, 1,1, t) \geq 0$ implies that $t=1$, and $\psi(t, t, 0,0, t) \leq$ 1 implies that $t=0$ for all $t>0$.

Then $A, B, S$ and $T$ have a unique common fixed point in $X$.

Proof Since the hybrid pairs $(A, S)$ and $(B, T)$ are OWC maps, there exist two elements $u, v \in X$ such that $A u \in S u, A S u \subseteq$ $S A u$ and $B v \in T v, B T v \subseteq T B v$.

First, we prove that $A u=B v$. As $A u \in S u$ and $B v \in T v$, so,

$$
\begin{aligned}
& M(A u, B v, t) \geq \delta_{M}(S u, T v, t), \\
& M(A u, T v, t) \geq \delta_{M}(S u, T v, t), \\
& M(B v, S u, t) \geq \delta_{M}(S u, T v, t), \\
& N(A u, B v, t) \leq \delta^{N}(S u, T v, t), \\
& N(A u, T v, t) \leq \delta^{N}(S u, T v, t), \\
& N(B v, S u, t) \leq \delta^{N}(S u, T v, t) .
\end{aligned}
$$

If $A u \neq B v$, then $\delta_{M}(S u, T v, t)<1$ and $\delta^{N}(S u, T v, t)>$ 0 . Using (1) for $x=u$ and $y=v$, we have

$$
\begin{gathered}
\phi\left\{\delta_{M}(S u, T v, t), M(A u, B v, t), 1,1,\right. \\
M(A u, T v, t) * M(S u, B v, t)\} \geq 0 \\
\psi\left\{\delta^{N}(S u, T v, t), N(A u, B v, t), 0,0,\right. \\
N(A u, T v, t) \diamond N(S u, B v, t)\} \leq 1 .
\end{gathered}
$$

That is,

$$
\begin{gathered}
\phi\left\{\delta_{M}(S u, T v, t), \delta_{M}(S u, T v, t)\right. \\
\left.1,1, \delta_{M}(S u, T v, t)\right\} \geq 0 \\
\psi\left\{\delta^{N}(S u, T v, t), \delta^{N}(A u, B v, t)\right. \\
\left.0,0, \delta^{N}(A u, T v, t)\right\} \leq 1 .
\end{gathered}
$$

Also, $\phi, \psi$ satisfies (b), so

$$
\delta_{M}(S u, T v, t)=1 \text { and } \delta^{N}(S u, T v, t)=0 .
$$

This is a contradiction which gives $A u=B v$

Now, we prove that $A^{2} u=A u$. Suppose that $A^{2} u \neq A u$, then $\delta_{M}(S A u, T v, t)<1$ and $\delta^{N}(S A u, T v t)>0$. Also, using (1) for $x=A u$ and $y=v$, we get

$$
\begin{gathered}
\phi\left\{\delta_{M}(S A u, T v, t), M(A A u, B v, t), 1,1,\right. \\
M(A A u, T v, t) * M(S A u, B v, t)\} \geq 0 \\
\psi\left\{\delta^{N}(S A u, T v, t), N(A A u, B v, t), 0,0,\right. \\
N(A A u, T v, t) \diamond N(S A u, B v, t)\} \leq 1 .
\end{gathered}
$$

Also, $A u \in S u$ and $A S u \in S A u$, so $A A u \in A S u \subseteq S A u$, $B v \in T v$ and $B T v \subseteq T B v$, hence

$$
\begin{aligned}
& M(A A u, B v, t) \geq \delta_{M}(S A u, T v, t), \\
& M(B v, S A u, t) \geq \delta_{M}(S A u, T v, t), \\
& N(A A u, B v, t) \leq \delta^{N}(S S A, T v, t), \\
& N(B v, S A u, t) \leq \delta^{N}(S A u, T v, t) .
\end{aligned}
$$

Therefore

$$
\begin{array}{r}
\phi\left\{\delta_{M}(S A u, T v, t), \delta_{M}(S A u, T v, t),\right. \\
\left.1,1, \delta_{M}(S A u, T v, t)\right\} \geq 0 \\
\psi\left\{\delta^{N}(S A u, T v, t), \delta^{N}(S A u, T v, t),\right. \\
\left.0,0, \delta^{N}(S A u, T v, t)\right\} \leq 1 .
\end{array}
$$

But $\phi, \psi$ satisfies (b), so,

$$
\delta_{M}(S A u, T v, t)=1 \text { and } \delta^{N}(S A u, T v, t)=0,
$$

a contradiction and hence $A^{2} u=A u=B v$. Similarly, we can show that $B^{2} v=B v$.

Let $A u=B v=z$, then $A z=z=B z, z \in S z$ and $z \in T z$. Therefore $z$ is a fixed point of $A, B, S$ and $T$.

Finally, we prove the uniqueness of the fixed point. Let $z \neq z_{0}$ be another fixed point of $A, B, S$ and $T$, then by (1), we have,

$$
\begin{gathered}
\phi\left\{\delta_{M}\left(S z, T z_{0}, t\right), \delta_{M}\left(A z, T z_{0}, t\right), 1,1,\right. \\
\left.\delta_{M}\left(A z, T z_{0}, t\right) * \delta_{M}\left(S z, T z_{0}, t\right)\right\} \geq 0 \\
\psi\left\{\delta^{N}\left(S z, T z_{0}, t\right), \delta^{N}\left(A z, T z_{0}, t\right), 0,0,\right. \\
\left.\delta^{N}\left(A z, T z_{0}, t\right) \diamond \delta^{N}\left(S z, T z_{0}, t\right)\right\} \leq 1 .
\end{gathered}
$$

From (b), we get

$$
\delta_{M}\left(S z, T z_{0}, t\right)=1, \quad \delta^{N}\left(S z, T z_{0}, t\right)=0 .
$$

This is a contradiction. Hence $z=z_{0}$. Therefore $z$ is unique common fixed point of $A, B, S$ and $T$. 
Example 3.2. Let $X$ be an IFMS in which $X=R^{+}, a * b=$ $\min \{a, b\}$ and $a \diamond b=\max \{a, b\}$ for all $a, b \in[0,1]$ such that for all $t>0$,

$$
M(x, y, t)=\frac{t}{t+d(x, y)}, \quad N(x, y, t)=\frac{d(x, y)}{t+d(x, y)} .
$$

Define the maps $A, B, S$ and $T$ on $X$ by

$$
\begin{aligned}
& A x= \begin{cases}2 x-1 & \text { if } x \leq 5, \\
2 x & \text { if } x>5,\end{cases} \\
& B x= \begin{cases}3-2 x & \text { if } x \leq 1, \\
x+1 & \text { if } 1>x,\end{cases} \\
& S x= \begin{cases}\{1\} & \text { if } x<2, \\
{[2 x, 2 x+5]} & \text { if } x \geq 2,\end{cases} \\
& T x= \begin{cases}\{1\} & \text { if } x=1, \\
{[x, x+2]} & \text { if otherwise. }\end{cases}
\end{aligned}
$$

Define $\phi:[0,1] \rightarrow[0,1], \psi:[0,1] \rightarrow[0,1]$ as

$$
\begin{aligned}
& \phi\left(t_{1}, t_{2}, t_{3}, t_{4}, t_{5}\right)=\min \left\{t_{1}, t_{2}, t_{3}, t_{4}, t_{5}\right\}, \\
& \psi\left(t_{1}, t_{2}, t_{3}, t_{4}, t_{5}\right)=\max \left\{t_{1}, t_{2}, t_{3}, t_{4}, t_{5}\right\} .
\end{aligned}
$$

Here the pairs $(A, S)$ and $(B, T)$ are OWC and the contractive condition is satisfied. Hence 1 is a unique common fixed point of $A, B, S$ and $T$.

Corollary 3.3. Let $X$ be an IFMS, $t * t=t$ and $t \diamond t=t$ for all $t \in[0,1]$ and let $A: X \rightarrow X$ and $S, T: X \rightarrow C B(X)$ be single and set-valued mappings such that the hybrid pair $(A, S)$ and $(A, T)$ are OWC satisfying

$$
\begin{gathered}
\phi\{M(S x, T y, t), M(A x, A y, t), \\
H(A x, S x, t), H(A y, T y, t), \\
H(A x, S y, t) * H(A y, S x, t)\} \geq 0 \\
\psi\{N(S x, T y, t), N(A x, A y, t), \\
h(A x, S x, t), h(A y, T y, t), \\
h(A x, S y, t) \diamond h(A y, S x, t)\} \leq 1
\end{gathered}
$$

for every $x, y \in X, t>0$ and $\phi, \psi$ are satisfies (a) and (b), respectively in Theorem 3.1. Then $A, S$ and $T$ have a unique common fixed point in $X$.

Proof Suppose that $A=B$ in Eq. (1) of Theorem 3.1, then we get this corollary.
Corollary 3.4. Let $X$ be an IFMS, $t * t=t$ and $t \diamond t=t$ for all $t \in[0,1]$ and let $A: X \rightarrow X$ and $S: X \rightarrow C B(X)$ be single and set-valued mappings such that the hybrid pair $(A, S)$ is OWC satisfying

$$
\begin{gathered}
\phi\left\{\delta_{M}(S x, S y, t), M(A x, A y, t),\right. \\
H(A x, S x, t), H(A y, S y, t), \\
H(A x, S y, t) * H(A y, S x, t)\} \geq 0 \\
\psi\left\{\delta^{N}(S x, S y, t), N(A x, A y, t),\right. \\
h(A x, S x, t), h(A y, S y, t), \\
h(A x, S y, t) \diamond h(A y, S x, t)\} \leq 1
\end{gathered}
$$

for every $x, y \in X, t>0$ and $\phi, \psi$ are functions satisfying (a) and (b), respectively in Theorem 3.1. Then $A$ and $S$ have a unique common fixed point in $X$.

Proof Suppose that $A=B$ and $S=T$ in Eq. (1) of Theorem 3.1 , then we get this corollary.

\section{Conclusion}

Park et.al. [8] introduced the IFMS, and proved common fixed point theorem in IFMS. Also, Park [9] proved some properties for several types compatible maps, and Park [10] defined occasionally weakly semi-compatible map and obtained some fixed point using this maps in IFMS.

In this paper, we introduce the notion of single and set-valued maps satisfying OWC property in IFMS using implicit relation. Also, we obtain common fixed point theorems for single and setvalued maps satisfying OWC property in IFMS using implicit relation.

\section{Conflict of Interest}

No potential conflict of interest relevant to this article was reported.

\section{Acknowledgments}

This author is supported by Chinju National University of Education Research Fund in 2014. 


\section{References}

[1] Z. Deng, "Fuzzy pseudo-metric spaces," Journal of Mathematical Analysis and Applications, vol. 86, no. 1, pp. 74-95, 1982. http://dx.doi.org/10.1016/0022-247X(82)90255-4

[2] M. Grabiec, "Fixed points in fuzzy metric spaces," Fuzzy Sets and Systems, vol. 27, no. 3, pp. 385-389, 1988. http: //dx.doi.org/10.1016/0165-0114(88)90064-4

[3] O. Kaleva and S. Seikkala, "On fuzzy metric spaces," Fuzzy Sets and Systems, vol. 12, no. 3, pp. 215-229, 1984. http: //dx.doi.org/10.1016/0165-0114(84)90069-1

[4] I. Kubiaczyk and S. Sharma, "Common coincidence point in fuzzy metric spaces," Journal of Fuzzy Mathematics, vol. 11 , no. 1, pp. 1-5, 2003.

[5] B. Singh and M. S. Chauhan, "Common fixed points of compatible maps in fuzzy metric spaces," Fuzzy Sets and Systems, vol. 115, no. 3, pp. 471-475, 2000. http://dx.doi. org/10.1016/S0165-0114(98)00099-2

[6] G. Jungck, "Compatible mappings and common fixed points," International Journal of Mathematics and Mathematical Sciences, vol. 9, no. 4, pp. 771-779, 1986. http: //dx.doi.org/10.1155/S0161171286000935

[7] P. Vijayaraju and Z. M. I. Sajath, "Common fixed points of single and multivalued maps in fuzzy metric spaces," Applied Mathematics, vol. 2, no. 5, pp. 595-599, 2011. http://dx.doi.org/10.4236/am.2011.25079

[8] J. H. Park, J. S. Park, and Y. C. Kwun, "A common fixed point theorem in the intuitionistic fuzzy metric space," in Proceedings of the 2nd International Conference on Advances in Natural Computation (ICNC) and 3rd International Conference on Fuzzy Systems and Knowledge Discovery (FSKD), Xian, China, 2006, pp. 293-300.
[9] J. S. Park, "Some properties for the compatible mappings in intuitionistic fuzzy metric space," Far East Journal of Mathematical Sciences, vol. 50, no. 1, pp. 79-86, 2011.

[10] J. S. Park, “On a common fixed point for occasionally weakly semi-compatible hybrid mappings in an intuitionistic fuzzy metric space," JP Journal of Fixed Point Theory and Applications, vol. 4, no. 1, pp. 1-10, 2009.

[11] B. Schweizer and A. Sklar, "Statistical metric spaces," Pacific Journal of Mathematics, vol. 10, no. 3, pp. 313-334, 1960.

[12] J. S. Park, "Fixed point theorems for weakly compatible functions using (JCLR) property in intuitionistic fuzzy metric space," International Journal of Fuzzy Logic and Intelligent Systems, vol. 12, no. 4, pp. 296-299, 2012. http://dx.doi.org/10.5391/IJFIS.2012.12.4.296

[13] J. S. Park, "Some common fixed point theorems using compatible maps in intuitionistic fuzzy metric space," International Journal of Fuzzy Logic and Intelligent Systems, vol. 11, no. 2, pp. 108-112, 2011. http://dx.doi.org/10.5391/ IJFIS.2011.11.2.108

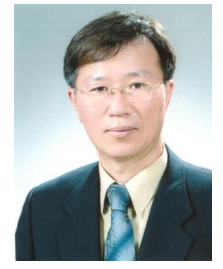

Jong Seo Park received the B.S., M.S. and Ph.D. degrees in mathematics from Dong-A University, Busan, Korea, in 1983, 1985 and 1995, respectively. He is currently Professor in Chinju National University of Education, Jinju, Korea. His research interests include fuzzy mathematics, fuzzy fixed point theory and fuzzy differential equation, etc.

E-mail: parkjs@cue.ac.kr 